\title{
Análisis de la distribución temática en el Boletín de la Sociedad Española de Cerámica y Vídrio
}

\author{
P. CUBERO ${ }^{A}$, O. SANZA , M. A. RODRÍGUEZ ${ }^{B}$ Y E. CRIADO \\ A Sociedad Española de Cerámica y Vidrio, C/ Kelsen 5, 28049 Madrid, España.

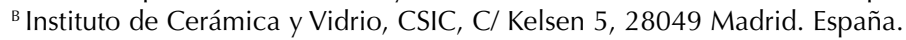

\begin{abstract}
Se ha realizado un análisis de la distribución temática de los trabajos publicados en el Boletín de la Sociedad Española de Cerámica y Vidrio. Se han analizado los trabajos comprendidos en el periodo 2003-2004. La distribución se ha realizado correlacionando la temática de los trabajos con las distintas secciones de la SECV. Esta asignación permite establecer una primera comparación con la relevancia del sector industrial de España. Se estudia la influencia que tienen estos parámetros sobre el factor de impacto de la revista.
\end{abstract}

Palabras clave: Cerámica, vidrio, factor impacto, revistas científicas.

Scope distribution analysis in the Bulletin of the Spanish Ceramic and Glass Society.

An analysis of the scope distribution of the papers published in Bulletin of the Spanish Ceramic and Glass Society has attempted. The papers analyzed has been those published in 2003-2004. The scope distribution has been realized by assigning the paper to the different section of the Spanish Ceramic and Glass Society. This assignation allows to compare this data with the industrial sector relevance. The influence of these parameters on the journal impact factor has also studied.

Keywords: Ceramic, glass, impact factor, scientific journals.

\section{INTRODUCCIÓN}

El Boletín de la Sociedad Española de Cerámica y Vidrio, SECV, cumple 44 años desde su aparición. Ésta se produjo ante la necesidad de difundir las actividades de una sociedad científica emergente y ha representado, sin duda, uno de los vehículos más eficaces en el desarrollo de la cerámica actual en España. En los primeros números de esta revista se pueden encontrar excelentes trabajos sobre materias primas, procesos, métodos de caracterización y aplicaciones que han conformado las bases de la ciencia y la tecnología en materiales cerámicos y vítreos en este país. Esos trabajos pioneros abordan prioritariamente aspectos diversos relacionados principalmente con la cerámica blanca y cerámica roja, pavimentos y revestimientos, refractarios y vidrios. De esta forma el Boletín de la SECV dio soporte científico y tecnológico a un amplio sector de actividades, muy ligados a industrias básicas y de la construcción, que estaban experimentando el paso de una industria manufacturera a otra mas modernizadas y con mayores exigencias tecnológicas. A lo largo de su historia puede decirse que el Boletín ha mantenido una relación dialéctica con las industrias cerámicas, contribuyendo a su desarrollo y al tiempo apoyándose en su extensión y apoyo económico para consolidar su posición en el mundo editorial

La evolución histórica de la ciencia y la tecnología de la cerámica y el vidrio ha dado lugar a la creación de diversos grupos de centros y especialidades en la mayor parte del territorio nacional. Los materiales cerámicos avanzados inicialmente y la actual implicación en Nanociencia constituyen las fuerzas conductoras de los científicos hacia nuevas áreas de conocimiento. El Boletín de la SECV sigue siendo una de las revistas claves para la difusión del conocimiento en cerámica y vidrio en lengua española. Esta posición se ha visto reforzada por su incorporación en 1998 al Institute of Scientific Information, ISI, que edita la clasificación de revistas científicas clasificadas por áreas de conocimiento y esta basada en el factor de impacto de las mismas, Scientific Citation Index, SCI. Recientemente (1) se han señalado las acciones necesarias para mejorar la calidad de los trabajos publicados en la revista. Aquellas acciones relacionadas con una mayor eficiencia de los procesos de evaluación y publicación ya se han abordado por el actual Comité de Redacción. Salvo de forma coyuntural (2-3) no se han realizado estudios con relación a la distribución de artículos por áreas temáticas, objeto que constituye el análisis que se realiza en este trabajo. Los cambios radicales que se están introduciendo en la industria cerámica y el cambio producido en el campo científico por la eclosión de la ciencia de materiales obligan a reflexionar sobre el futuro de publicaciones como el Boletín de la SECV.

\section{DISTRIBUCIÓN TEMÁTICA DE TRABAJOS PUBLICADOS.}

Se han empleado para este análisis los trabajos publicados en los años 2003 y 2004, analizándose un total de 234 artículos. Esta cantidad representa un número muy elevado de artículos si se compara con los publicados en un periodo equivalente anterior, 2001-2002, que fueron 126 en total. Este aumento en 
TABLA I. DISTRIBUCIÓN DE TRABAJOS EN CERÁMICA Y VIDRIO PUBLICADOS EN EL BOLETÍN DE LA SECV EN 2003-2004

\begin{tabular}{|c|c|c|c|c|c|}
\hline & $\mathrm{N}^{\mathrm{o}}$ Artículos & Promedio de autores & Promedio grupos & $\%$ empresas en & $\%$ grupos extranjeros \\
\hline & & por artículo & por artículo & esos grupos & \\
\hline ARTE Y DISEÑO & 3 & 3,33 & 1,33 & 0,00 & 0,00 \\
\hline ELECTROCERAMICA & 50 & 5,12 & 1,9 & 12,94 & 45,88 \\
\hline $\begin{array}{l}\text { ESMALTES Y } \\
\text { PIGMENTOS } \\
\text { CERAMICOS } \\
\end{array}$ & 4 & 4,5 & 1,75 & 42,86 & 28,57 \\
\hline LADRILLOS Y TEJAS & 0 & 0 & 0,00 & 0,00 & 0,00 \\
\hline MATERIAS PRIMAS & 15 & 3,93 & 2,47 & 24,32 & 8,11 \\
\hline MEDIO AMBIENTE & 7 & 4,14 & 1,29 & 0 & 11,11 \\
\hline $\begin{array}{l}\text { PAVIMENTOS Y } \\
\text { REVESTIMENTOS } \\
\text { CERAMICOS } \\
\end{array}$ & 7 & 3,71 & 2,14 & 6,67 & 46,67 \\
\hline REFRACTARIOS & 9 & 4,56 & 2,33 & 23,81 & 42,86 \\
\hline
\end{tabular}

TABLA II. DISTRIBUCIÓN TEMÁTICA DE LOS TRABAJOS PUBLICADOS EN LAS SECCIONES DE CIENCIA BÁSICA Y ELECTROCERÁMICA.

\begin{tabular}{|c|c|c|c|c|}
\hline Sección & Temática & $\mathrm{N}^{\mathrm{o}}$ Artículos & $\begin{array}{l}\text { Promedio de autores por } \\
\text { artículo }\end{array}$ & Promedio grupos por articulo \\
\hline \multirow[t]{7}{*}{ CIENCIA BASICA } & Avanzados & 15 & 5 & 1,53 \\
\hline & Nanomateriales & 4 & 4,25 & 1,50 \\
\hline & Biocerámicas & 4 & 4 & 1,50 \\
\hline & Proceso y Conformado & 5 & 4 & 1,40 \\
\hline & Recubrimientos y membranas & 6 & 4,17 & 2 \\
\hline & Otros & 2 & 2 & 2 \\
\hline & Total & 36 & 4,44 & 1,61 \\
\hline \multirow[t]{5}{*}{ ELECTROCERAMICA } & Conductores & 20 & 4,30 & 1,70 \\
\hline & Ferroeléctricos & 17 & 4,53 & 1,41 \\
\hline & Dieléctricos & 4 & 3,75 & 1,50 \\
\hline & Magnéticos & 6 & 6,00 & 2,33 \\
\hline & Sensores & 3 & 7 & 2,33 \\
\hline
\end{tabular}

TABLA III. DISTRIBUCIÓN DE TRABAJOS DEL Nº ESPECIAL DE MATERIALES.

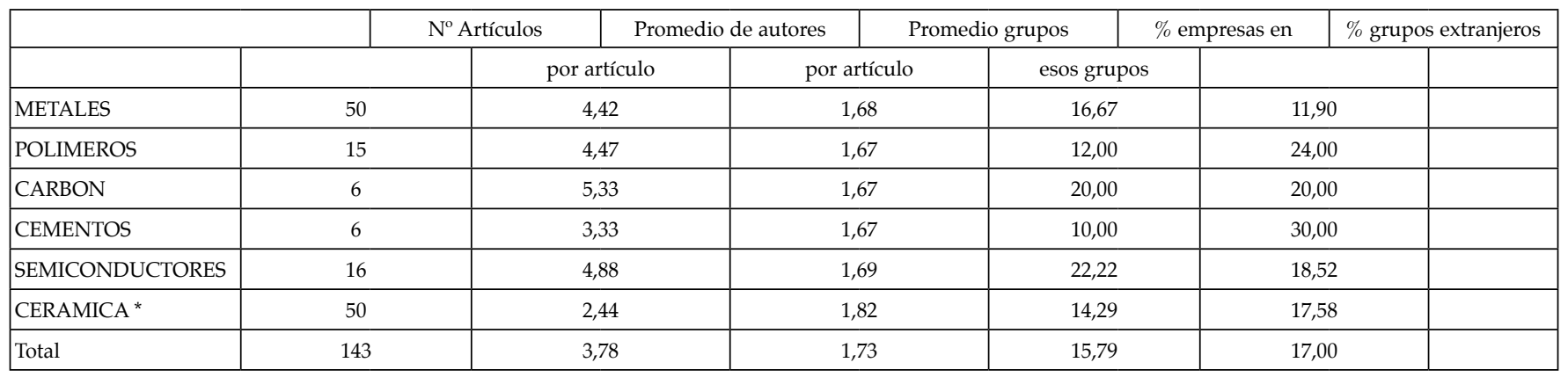

* Estos trabajos han sido incluidos también en la tabla I.

número de artículos se debe en gran medida a la publicación de 143 trabajos presentados en el VII Reunión Nacional de Materiales que se celebró en Madrid en el 2002.

La distribución de estos artículos por áreas temáticas relacionadas con Cerámica y Vidrio se realizó asignando los trabajos publicados a las diferentes secciones que conforman la SECV. Lógicamente, un buen número de trabajos se pueden encuadrar en más de una sección, en este caso la asignación se realizó atendiendo al objetivo principal del trabajo. La tabla I y II recogen la distribución de los mismos. La distribución temática de los trabajos publicados en relación con la VII Reunión Nacional de Materiales se resume en la Tabla III.

La distribución de trabajos por secciones muestra que las secciones donde se produce un mayor número de publicaciones son las de Electrocerámica, 50 trabajos, y Ciencia Básica. 36. Si se comparan estos resultados con los datos de producción de la industria española de la cerámica y el vidrio (4) se puede apreciar una disociación entre la realidad industrial y el interés científico.Este mismo desencuentro se detectaba ya cuando se analizaba la comparación entre los proyectos de investigación básicos aprobados por el plan Nacional de Investigación y los proyectos de desarrollo tecnológico financiados por el Centro de Desarrollo Tecnológico e Industrial (CDTI) o los contratos de investigación entre empresas y organismos públicos de investigación (3). Se puede argumentar que precisamente del avance científico deberían surgir las nuevas 
iniciativas empresariales. La incipiente industria nacional en Electrocerámica y en materiales cerámicos avanzados con aparición de varias empresas en los últimos años parece un buen aval de futuro. Pero no obstante la labor de generación de conocimiento básico no será efectiva si no se realiza la adecuada labor de difusión y el correspondiente acercamiento a las industrias por parte de los científicos y tecnólogos. Esta labor debe ser correspondida por los sectores empresariales que deberían considerar las nuevas tecnologías como elemento de futuro en sus sistemas productivos. Por otra parte, el sistema de ciencia y tecnología español con un predominio de la inversión en I+D del sector público, fomenta en gran medida esta disociación de intereses.

El promedio de autores por artículo es bastante homogéneo en las diferentes secciones, siendo de 4.4 autores por artículo. El número de grupos que hacen posible un trabajo es de 1.85 grupos por trabajo en promedio, aspecto que indica un fuerte componente de colaboración entre diferentes grupos, en su mayoría con un carácter complementario. Las secciones con un mayor número de artículos son aquellas que presentan un menor promedio de grupos por artículo. Mientras que un mayor número de grupos puede indicar una orientación más aplicada y multidisciplinar de los trabajos, un menor número de grupos parecería estar justificando trabajos orientados hacia ciencia básica. El porcentaje de grupos con origen empresarial o privado es en promedio cercano al $17 \%$ e indica la baja participación empresarial en las actividades de divulgación de resultados. Destaca la presencia de cerca de un $43 \%$ de empresas en los trabajos relacionados con esmaltes y pigmentos cerámicos debida a la relevancia que las empresas españolas tienen en este sector y fundamentada en grupos con estructuras de $\mathrm{I}+\mathrm{D}+\mathrm{i}$ consolidadas, si bien el volumen de estos trabajos es poco significativo.

Un aspecto que demuestra la internacionalización de la revista es el alto número de grupos extranjeros que publica sus trabajos en la revista ya que 1 de cada 3 grupos lo es. El origen de estos grupos es fundamentalmente Iberoamericano, aunque se constata un aumento progresivo de contribuciones europeas. Las secciones que destacan por la alta presencia de grupos extranjeros son pavimentos y revestimientos cerámicos, electrocerámica, refractarios y vidrios, las cuales superan el $40 \%$ de grupos extranjeros. El hecho de que en los sectores con fuerte industrialización en España se produzca un mayor número de publicaciones por parte de grupos extranjeros señalaría que los grupos españoles, en general, no contemplan entre sus líneas de trabajo realizar avances científicos o tecnológicos en sectores maduros como corresponde a pavimentos y revestimientos, refractarios y vidrios. En el caso de la sección de Electrocerámica, la publicación bianual de los trabajos presentados en la reunión nacional ha incorporado un creciente número de autores extranjeros a publicar regularmente trabajos en el Boletín de la SECV.

La distribución de trabajos correspondiente al número especial de materiales muestra que cerca de un $39 \%$ de trabajos, esto es 93 trabajos sobre un total de 243 en el periodo en estudio, se publicaron en áreas de conocimiento que no están relacionadas con la cerámica y el vidrio. Como caso extremo, se constata que se publicaron 50 trabajos sobre metales a pesar de existir una revista española de reconocido prestigio especializada en metales, Revista de Metalurgia.

\section{TEMATICA DEL BOLETÍN SECV.}

En la sección de Electrocerámica el mayor volumen de trabajos se centra en diferentes tipos de conductores. Se han reportado trabajos relacionados con la síntesis y caracterización de conductores catiónicos e iónicos de estado sólido (3-8), conductores mixtos (11-13), protónicos (14). Así mismo, y dado el fuerte interés que este tipo de materiales presentan en la obtención de energía, se han publicado trabajos relacionados con los diferentes materiales cerámicos como son electrolitos sólidos (15), y electrodos cerámicos para pilas y baterías (16-21). El aumento de este tipo de trabajos se debe a los esfuerzos en las redes de I+D nacionales y transnacionales en pilas de combustible en las que participan empresas del sector energético y donde las empresas cerámicas españolas tienen poca presencia.

Los materiales ferroeléctricos constituyen el otro gran bloque temático dentro de la sección de electrocerámica, estando centrados en mayor número en las propiedades de estos materiales conformados en forma de lámina delgada (22-26) debido al fuerte interés europeo sobre la integración de dichos dispositivos en sistemas electrónicos, si bien no existen empresas nacionales de microelectrónica que puedan ser beneficiarias de este tipo de información; el estudio de fenómenos no lineales de piezoeléctricos masivos (27-28), los nuevos materiales y sus propiedades (29-35) y las aplicaciones de dichos materiales (36-38), destacando en particular aquellas destinadas a la biomecánica debido al nacimiento de empresas en este sector como resultados de los procesos spin-off del Instituto de Automática industrial del CSIC. Los avances en cerámicas dieléctricas se han centrado en varistores cerámicos (39-41), estos trabajos presentan relación con la reciente

TABLA IV . CLASIFICACIÓN DEL AÑO 2004 POR FACTOR DE IMPACTO DE LA CATEGORÍA CIENCIA DE MATERIALES: CERÁMICA

\begin{tabular}{|c|c|c|c|}
\hline Posición & Revista & $\begin{array}{l}\text { Citas } \\
\text { totales }\end{array}$ & $\begin{array}{l}\text { Factor } \\
\text { impacto }\end{array}$ \\
\hline 1 & J AM CERAM SOC & 23099 & 1,710 \\
\hline 2 & J EUR CERAM SOC & 5243 & 1,483 \\
\hline 3 & J NON-CRYST SOLIDS & 15203 & 1,433 \\
\hline 4 & J SOL-GEL SCI TECHN & 1912 & 1,150 \\
\hline 5 & J ELECTROCERAM & 487 & 1,124 \\
\hline 6 & CERAM INT & 1403 & 1,040 \\
\hline 7 & PHYS CHEM GLASSES & 1463 & 0,727 \\
\hline 8 & J CERAM SOC JPN & 1472 & 0,608 \\
\hline 9 & J CERAM PROCESS RES & 76 & 0,470 \\
\hline 10 & AM CERAM SOC BULL & 2031 & 0,401 \\
\hline 11 & CERAM-SILIKATY & 78 & 0,385 \\
\hline 12 & GLASS TECHNOL & 302 & 0,347 \\
\hline 13 & BRIT CERAM T & 622 & 0,330 \\
\hline 14 & BOL SOC ESP CERAM V & 155 & 0,310 \\
\hline 15 & KEY ENG MATER & 1803 & 0,278 \\
\hline 16 & IND CERAM & 95 & 0,273 \\
\hline 17 & GLASS PHYS CHEM+ & 218 & 0,261 \\
\hline 18 & GLASS CERAM+ & 266 & 0,244 \\
\hline 19 & GLASS SCI TECHNOL & 223 & 0,241 \\
\hline 20 & J INORG MATER & 411 & 0,223 \\
\hline 21 & REFRACT IND CERAM+ & 101 & 0,156 \\
\hline 22 & POWDER MATALL MET C+ & 339 & 0,148 \\
\hline 23 & MATER WORLD & 105 & 0,115 \\
\hline 24 & SILIC IND & 79 & 0,065 \\
\hline 25 & CFI-CERAM FORUM INT & 90 & 0,057 \\
\hline
\end{tabular}


TABLA V. NÚMERO DE VECES QUE UN ARTÍCULO DE LA REVISTA REFERIDA SE CITA EN EL BOLETÍN DE LA SECV.

\begin{tabular}{|c|l|c|}
\hline $\begin{array}{c}\text { Factor de } \\
\text { impacto }\end{array}$ & Revista & $\mathrm{N}^{\circ}$ citas \\
\hline 1,710 & J AM CERAM SOC & 99 \\
\hline 1,862 & SOLID STATE IONICS & 80 \\
\hline 2,255 & J APPL PHYS & 68 \\
\hline 0,864 & J MATER SCI & 57 \\
\hline 3,075 & PHYS REV B & 57 \\
\hline 0,310 & BOL SOC ESP CERAM V & 51 \\
\hline 1,483 & J EUR CERAM SOC & 48 \\
\hline 4,308 & APPL PHYS LETT & 40 \\
\hline 1,815 & J SOLID STATE CHEM & 35 \\
\hline 2,108 & J MEMBRANE SCI & 33 \\
\hline 2,721 & J MATER CHEM & 32 \\
\hline 1,433 & J NON-CRYST SOLIDS & 32 \\
\hline 2,356 & J ELECTROCHEM SOC & 30 \\
\hline 7,218 & PHYS REV LETT & 29 \\
\hline 1,647 & THIN SOLID FILMS & 29 \\
\hline 1,445 & MAT SCI ENG A-STRUCT & 26 \\
\hline 1,310 & MATER RES BULL & 22 \\
\hline 4,103 & CHEM MATER & 17 \\
\hline 1,432 & SURF COAT TECH & \\
\hline
\end{tabular}

implantación de este tipo de industria en España, derivados de procesos de transferencia de tecnología desde el Instituto de Cerámica y Vidrio, CSIC. También se ha publicado un interesante trabajo sobre nuevos materiales y metodologías para substratos que incorporen funcionalidades (42). Un número creciente de trabajos incorporados al Boletín los relacionados con relación con las propiedades magnéticas de cerámicas con estructura espinela (42-43) y perovskita (44-47) entre los que cabe destacar el interesante trabajo de Fontcuberta y colaboradores sobre avances recientes en cerámicas magnetoestrictivas (44). En el apartado de sensores las publicaciones estuvieron relacionadas con la obtención y caracterización de sensores de gases (48-50).

La sección de Ciencia Básica concentra un buen número de trabajos que abordan temas diferentes. La mayoría de estos se centra en el ámbito de los materiales cerámicos avanzados en los que se abordan métodos de obtención (51-53), influencia de las composiciones en las propiedades (54-57), su comportamiento en condiciones extremas (58-61) y un importante número de trabajos en recubrimientos y membranas (62-71). En los aspectos de procesado y conformado se presenta un reducido número de trabajos que abordan procesos de tratamiento láser para texturación (72) o corte (73). Tratamientos en horno solar (74) o conformado de piezas mediante el empleo de suspensiones parafínicas (75).

En biocerámicas y biovidrios la atención se enfocó en la bioactividad de materiales vitrocerámicos (76) y en recubrimientos sobre aleaciones metálicas (77-79).

Un reducido número de trabajos relacionados con nanotecnología (80-83) demuestra que las publicaciones en esta disciplina están o bien alejada del interés de los grupos que trabajan en cerámica y vidrio, o bien, dichas publicaciones se dirigen a revistas más especializadas y de mayor factor de impacto. Ambos aspectos son altamente preocupantes, aunque el más grave sea la posible pérdida de posicionamiento del sector de cerámica y vidrio frente a nuevos desarrollos y oportunidades.

En la sección de refractarios destaca por su interés práctico la publicación del diagrama de equilibrio de fases correspondiente al sistema cuaternario $\mathrm{Al}_{2} \mathrm{O}_{3}-\mathrm{CaO}-\mathrm{MgO}-\mathrm{SiO}_{2}$ (84) y los estudios de cementos refractarios objeto de un proceso de transferencia de tecnología desde el Instituto de Cerámica y Vidrio, CSIC, a la empresa Refractarios Alfrán S.A. culminado con éxito (85-87). Así mismo se han publicado trabajos en relación a otros materiales refractarios y sus propiedades (88-90) y desarrollo de cuerpos porosos por espumado (91).

En la sección de materias primas la atención se ha puesto en las propiedades luminiscentes de distintas materias primas y residuos para cerámica (92-98), destacando el estudio sobre el circón radiactivo a partir de su curva termoluminiscente (92) por aportar un método de caracterización para estos

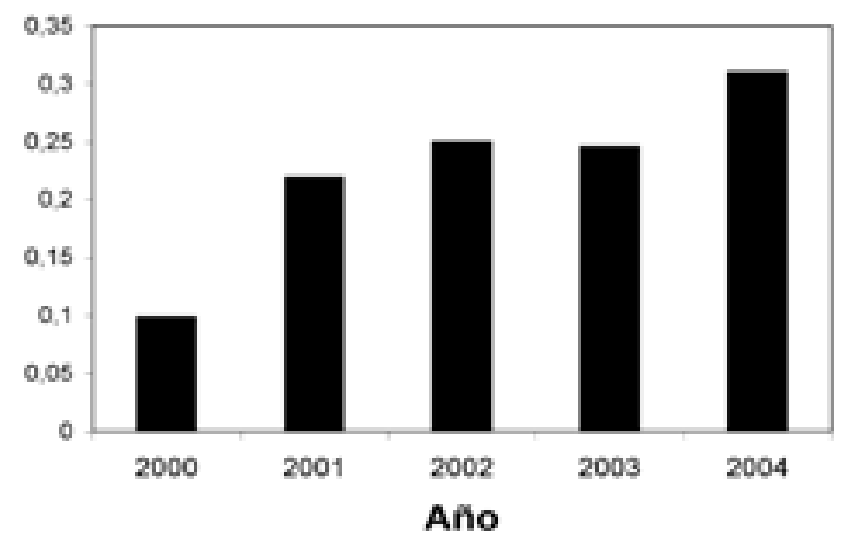

Figura 1. Evolución del Factor de impacto SCI del Boletín de la Sociedad Española de Cerámica y Vidrio.

materiales de fuerte interés en el sector de pavimentos y revestimientos. El resto de los trabajos se centraron en la caracterización y propiedades de diferentes yacimientos y residuos (99-102) y obtención de materias primas avanzadas con interés tecnológico (103-106).

En la sección de vidrios el tema preferente de atención se centró en los procesos sol-gel para obtención de circón en el sistema alúmina-circona-sílice (107), recubrimientos con propiedades ópticas (108-109), materiales híbridos (110) y metodologías para la obtención de materiales por proceso solgel (111-113). Destaca el trabajo presentado en sellos vítreos y vitrocerámicos para pilas de combustible de óxido sólido por su alta componente de aplicación (114). Se constata una fuerte separación entre la temática de estos trabajos y el fuerte sector industrial vidriero.

En la emergente sección de Medio Ambiente se recogieron materiales con aplicaciones e intereses muy diversas. La fuerte preocupación medioambiental del sector cerámico se reflejo sobre la evolución, estrategias y resultados de la investigación medioambiental en la producción de baldosas cerámicas (115). Se han reportado trabajos sobre el comportamiento de materiales cerámicos y vítreos como absorbentes de metales pesados en aguas industriales (116) o soportes activos para la biorremediación (117), retención de gases contaminantes (118), deshidratación de disolventes industriales (119), inmovilización de biocatalizadores (120), biorremediación de metales (120) e influencia de la radiación microondas en la estructura de materiales laminares (121).

En pavimentos y revestimientos los trabajos publicados abordaron influencias de diferentes feldespatos en las propiedades reológicas de las barbotinas (122); determinación de propiedades de interés tecnológico en baldosas (123-125); estudio de la distribución de tensión durante la cocción (126- 
127), y análisis del sistema de operaciones del sector cerámico español (128).

En esmaltes y pigmentos cerámicos se publicaron nuevas metodologías para el desarrollo de vidriados vitrocerámicos (129) y pigmentos (130-132).

La temática de arqueomometría que se ubica en la sección de arte y diseño presentó trabajos sobre caracterización de materiales cerámicos (133-134) y vidrios (135).

\section{IMPACTO DEL BOLETÍN SECV}

El impacto científico de una revista se determina siguiendo la clasificación de ISI en el SCI. La forma de calcularlo es dividiendo el número de citas de la revista recogidas a lo largo de un año y referidas a los artículos publicados en los dos años anteriores. Este número de citas se divide entre el número de artículos publicados en esos dos años. A modo de ejemplo, el factor de impacto del Boletín SECV en 2004 fue de 0.310 , siendo el resultado de recibir en 2004 la cantidad de 35 citas (7 de artículos publicados en 2003 y 28 de artículos publicados en 2002) entre 113 artículos publicados (40 en 2003 y 73 en 2002). Este factor de impacto supone un ligero incremento en referencia a años anteriores, figura 1.

En el contexto del área de conocimiento donde se encuadra la revista, Ciencia de Materiales, la revista Bol. Soc. Esp. Ceram. V. ocupa en la posición 14 de un total de 25. Esta posición no es una posición estable pues pequeñas variaciones en el número de citas se traducirían en una fuerte variación en posición. En otros términos, si todos los trabajos que se publican en la revista recibieran al menos una cita en los dos años posteriores, la posición de la revista se consolidaría entre las diez primeras, siendo ésta una labor que los mismos autores pueden asumir.

Respecto a la procedencia de las citas, el $30 \%$ se producen en la misma revista y sólo hay dos revistas que aporten casi un $10 \%$ de citas respectivamente, estas son: J. Eur. Cer. Soc. y Key Eng. Mat.. El resto de citas se toman de forma dispersa en muchas otras revistas.

Un dato interesante son las fuentes que se citan en los artículos publicados en el Boletín de la SECV. La tabla V presenta las 20 revistas más citadas por el Boletín (de un total de 270 ). El propio Boletín ocupa la $6^{\mathrm{a}}$ posición y cabe destacar que en el resto de revistas, las fuentes corresponden a trabajos publicados en revistas de factores de impacto elevados o muy elevados. Este aspecto señalaría una calidad de las temáticas alta y lleva a la siguiente reflexión: ¿Por qué el Boletín de la SECV recibe pocas citas?. La respuesta parece estar asociada a la misma naturaleza de la publicación de trabajos científicos en lengua española que constituye una fuerte limitación en cuanto a la difusión. En este periodo el 10,6\% de los trabajos de la revista se publicaron en ingles. En los trabajos de cerámica y vidrio este porcentaje sube hasta aproximadamente el 15\%, mientras que los trabajos en otras áreas derivados del número especial de materiales solo se publicó un 4,3\% en ingles. En este aspecto destaca que el $32 \%$ los trabajos publicados en la sección de elctrocerámica lo fue en ingles.

Uno de los aspectos más limitantes para que el factor de impacto aumente es la publicación de trabajos ajenos al ámbito de la cerámica y el vidrio. En este sentido el número especial de materiales con un $40 \%$ del total de los trabajos en áreas diferentes contribuirá a corto plazo a una severa disminución de dicho factor de impacto.

El análisis de la evolución del futuro de las revistas científicas en España incluidas e en el S.C.I. ha sido fruto de diversos análisis (136) que reflejan los múltiples factores que afectan a la variabilidad de su factor de impacto y las dificultades que existen para su propia viabilidad a medio plazo. Para lograr mejorar los factores de impacto serán necesarios no sólo un cambio en la cultura de los científicos españoles, que citan poco los trabajos que publican en revistas nacionales, sino un incremento de los apoyos económicos y mejora de su difusión en los portales informáticos, que contribuyan a mejorar su visibilidad internacional

\section{CONCLUSIONES}

Se ha analizado la distribución temática de los trabajos publicados en la revista Boletín de la Sociedad Española de Cerámica y Vidrio en el periodo 2003-2004 derivándose las siguientes conclusiones:

- En interés científico de las publicaciones difiere en gran medida de los intereses productivos de la industria de la cerámica y el vidrio en España.

- La mejora del impacto científico de la revista determinado como factor de impacto en la clasificación SCI, esta fuertemente limitada a corto plazo por la publicación de trabajos ajenos al ámbito de la cerámica y el vidrio, y en un medio plazo por el idioma.

- Se ha producido una internacionalización en la procedencia de los autores que contribuyen con trabajos.

\section{AGRADECIMIENTOS}

Los autores agradecen al Comité de Redacción de la revista y en particular a J. F. Fernández por su colaboración y comentarios en la realización de este trabajo.

\section{BIBLIOGRAFÍA}

1. E. Criado. "El Boletín de la Sociedad Española de Cerámica y Vidrio" Bol. Soc. Esp. Ceram. V. 42, 6, 397-401 (2003)

2. Vazquez, M, Galbán C. " Estudio bibliométrico de la Revista Boletín de la Sociedad Española de Cerámica y Vidrio “. Bol Soc. Esp. Ceram. V. 28, 5, 327-333 (1984)

3. E.Criado, J. Gonzalez Ayuso, A. Durán. “ Programa Nacional de Materiales 1988-92. Una primera evaluación". Bol. Soc .Esp. Ceram. V. 32, 3, 191-208 (1993)

4. E. Criado, E. Sanchez, M. Regueiro. "La industria cerámica española, ¿ante un cambio de ciclo?" Bol. Soc. Esp. Ceram. V. 43, 1, 85-101 (2004)

5. O. A. Smirnova, V. V. Kharton, F. M. B. Marques. "Synthesis and characterization of sodium cation-conducting $\mathrm{Na}\left(\mathrm{M}_{\mathrm{L}}\right) \mathrm{O}_{2}\left(\mathrm{M}=\mathrm{Ni}^{2+}, \mathrm{Fe}^{3+} ; \mathrm{L}=\mathrm{Ti}^{4+}, \mathrm{Sb}^{5+}\right)^{\prime \prime}$. Bol. Soc. Esp. Ceram. V. 43, 3, 679-685 (2004)

6. R. O. Fuentes, D. G. Lamas, M. E. Fernández de Rapp, F. M. Figueiredo, J. R. Frade, F. M. B. Marques, J. I. Franco. "Restrictions to obtain NASICON by a ceramic route". Bol. Soc. Esp. Ceram. V. 43, 4, 775-779 (2004)

7. V. Peña, A. Rivera, J. García-Barriocanal, C. León, J. Santamaría, E. GarcíaGonzalez, J. M. González-Calbet. "Conductividad eléctrica y difusión de oxígeno en el sistema Bifevox". Bol. Soc. Esp. Ceram. V. 43, 1, 67-70 (2004)

8. J. Mata, X. Solans, M. Font-Bardia . "Estudio del Sistema $\mathrm{Li}_{2} \mathrm{SO}_{4}-\mathrm{Na}_{2} \mathrm{SO}_{4}$. Diagrama de fases y caracterización del $\mathrm{LiNaSO}_{4}$ ". Bol. Soc. Esp. Ceram. V. 43, 4, 810-814 (2004)

9. K. J. Moreno, G. Mendoza-Suárez, A. F. Fuentes, J. García-Barriocanal, C. León, J. Santamaría. "Movilidad de oxígeno en conductores iónicos $\mathrm{A}_{2} \mathrm{Ti}_{2-\mathrm{y}} \mathrm{Zr}_{\mathrm{y}} \mathrm{O}_{7}(\mathrm{~A}$ : Y,Gd)". Bol. Soc. Esp. Ceram. V. 43, 4, 759-763 (2004)

10. G. García, J. Caro, J. Santiso, J. A. Pardo, A. Figueras, A. Abrutis."Crecimiento de películas delgadas de membranas de conducción iónica mediante la técnica PIMOCVD". Bol. Soc. Esp. Ceram. V. 43, 2, 448-451 (2004)

11. J. R. Frade, V. V. Kharton, A. A. Yaremchenko, E. V. Tsipis, A. L. Shaula, E. N. Naumovich, A. V. Kovalevsky, F. M. B. Marques. "Mixed conducting materials for partial oxidation of hydrocarbons". Bol. Soc. Esp. Ceram. V. 43, 3, 640-643 (2004) 
12. G. C. Mather, J. R. Jurado. "Nonstoichiometry and stability in water of undoped $\mathrm{SrCeO}^{\prime \prime}$. Bol. Soc. Esp. Ceram. V. 42, 5, 311-316 (2003)

13. L. Mestre, M. L. Martínez-Sarrión, F. J. Lepe. “Optimización de un nuevo método de preparación de óxidos mixtos tipo perovskita con vacantes de oxígeno". Bol. Soc. Esp. Ceram. V. 43, 4, 764-768 (2004)

14. M. J. Lopez Robledo, A. R. Pinto Gómez, J. Martínez Fernández, A. R. De Arellano López, A. Sayir. "Microestructura y propiedades mecánicas de conductores protónicos de alta temperatura crecidos por fusión de zona flotante". Bol. Soc. Esp. Ceram. V. 43, 4, 753-758 (2004)

15. J. A. Bademes. P. Benet, S. Sorli, M. A. Tena, G. Monrós. " Characterisation of Y-PSZ and PR-doped Y-PSZ obtained by unconventional methods for sofc applications". Bol. Soc. Esp. Ceram. V. 43, 4, 787-791 (2004)

16. J. M. Amarilla, R.M. Rojas, M. L. Perez-Revenga, M.G. Lazarraga, S. Mandal, J.M. Rojo. “Optimización de espinelas LiCoyMn $\mathrm{M}_{2}-\mathrm{yO}_{4}$ para electrodos positivos de baterías recargables de ion-litio mediante ajuste del dopado y de la temperatura de síntesis". Bol. Soc. Esp. Ceram. V. 43, 2, 127-131 (2004)

17. J. C. Rendón, L. M. Valacez, J.L. Frodriguez, J. Mendez, J.Lopez. "Síntesis y sinterización de soluciones sólidas de cromita de lantano obtenidas por el método de coprecipitación en medio alcalino". Bol. Soc. Esp. Ceram. V. 43, 5, 869-876 (2004)

18. M. Sanchez, S. Yañez, A. Castro, S. Castro, M. A. Señaris. "Preparación y caracterización de óxidos de cobalto Cuasi-bidimensionales $\mathrm{Ln}_{1-\mathrm{Sr}_{1+\times} \mathrm{CoO}}$ (Ln=La, Nd y Gd)". Bol. Soc. Esp. Ceram. V. 43, 4, 748-752 (2004)

19. A. A. Yaremchenko, A. L. Shaula, V. V. Kharton, A. V. Kovalevsky, E. N. Naumovich, J. R. Frade, F. M. B. Marques. "Oxygen permeability of transition metal-containing $\mathrm{La}(\mathrm{Sr}, \mathrm{Pr}) \mathrm{Ga}(\mathrm{Mg}) \mathrm{O}_{3-}$ ceramic membranes. Bol. Soc. Esp. Ceram. V. 43, 4, 769-774 (2004)

20. S. Castro-García, M. A. Señaris, A. Castro, C. Julien. “Influencia de la síntesis y del grado de dopaje en las propiedades morfológicas, estructurales y electroquímicas de óxidos $\mathrm{LiCo}_{1-\mathrm{x}} \mathrm{M}_{\mathrm{x}} \mathrm{O}_{2}(\mathrm{M}=\mathrm{Ni}, \mathrm{Al}, \mathrm{Mg})$. Bol. Soc. Esp. Ceram. V. 43, 4, 780-786 (2004)

21. M. P. Gutierrez, B. Rivas, M. A. Señaris, J. Rivas, “Caracterización dieléctrica de la perovskita laminar $\mathrm{La}_{1,5} \mathrm{Sr}_{0,5} \mathrm{CoO}_{4}$ ". Bol. Soc. Esp. Ceram. V. 43, 3, 649-652 (2004)

22. M. H. Lente, A. L. Bacichetti, R. G. Mendes And J. A. Eiras. "Nonlinear dielectric response in ferroelectric thin films". Bol. Soc. Esp. Ceram. V. 43, 4, 815-818 (2004)

23. P. Quintana, A. I. Olivia, F. Alonzo, J. E. Corona, W. Cauich. “Evolución microestructural de capas delgadas de cobre por flujo de corriente eléctrica aplicada". Bol. Soc. Esp. Ceram. V. 43, 4, 805-809 (2004)

24. J. Mendiola, C. Alemany, R. Jimenez, E. Maurer. M. L. Calzada. "PbTiO, modificado con Ca: de la cerámica a la lámina". Bol. Soc. Esp. Ceram. V. 43, 3, 620-626 (2004)

25. J Mendiola, R. Jimenez, P. Ramos, C. Alemany, M. L. Calzada, E.Maurer. "Propiedades dieléctricas de laminas delgadas de $(\mathrm{Pb}, \mathrm{Ca}) \mathrm{TiO}_{3}$ con alto contenido de Ca". Bol. Soc. Esp. Ceram. V. 43, 2, 445-447 (2004)

26. R. Gonzalez-Ballesteros, E. Suaste-Gómez. “Determinación del coeficiente piroeléctrico del sistema ferroeléctrico cerámico de $\mathrm{Pb}_{0,88} \mathrm{Ln}_{0,08} \mathrm{Mn}_{0,02} \mathrm{O}_{3}(\mathrm{Ln}=\mathrm{La}$, $\mathrm{Sm}, \mathrm{Eu}$ ) y su aplicación en detectores de infrarrojo". Bol. Soc. Esp. Ceram. V. 43, 6, 915-918 (2004)

27. A. Albareda, R. Pérez, J.E García, J. A. Casals, M. Udina. “No linealidad del comportamiento elástico de las cerámicas piezoeléctricas". Bol. Soc. Esp. Ceram. V. 43, 2, 119-126 (2004)

28. R. Pérez, A. Albareda, J.E. García, J. A. Casals. “Relación entre los comportamientos no lineales dieléctrico y mecánico en cerámicas piezoeléctricas de PZT". Bol. Soc. Esp. Ceram. V. 43, 3, 658-662 (2004)

29. S. R. De Lazaro, E. R. Leite, E. Longo, A. Beltrán. "Fotoluminiscencia del $\mathrm{PbTiO}_{3}$ en estado amorfo analizada por métodos ab-initio periódicos". Bol. Soc. Esp. Ceram. V. 43, 3, 644-648 (2004)

30. L. Pardo, B. Jiménez, T. Hungría, A. Moure, A. Castro. "Sodium-lithium niobate piezoceramics prepared by mechanochemical activation assisted methos". Bol. Soc. Esp. Ceram. V. 43, 1, 30-34 (2004)

31. A. A. Cavalheiro, M. A. Zaghete, M. Cilense, M. Villegas, J. F. Fernandez, J. A. Varela. "Estudio de la adición de $\mathrm{K}^{+}$y $\mathrm{LiNbO}_{3}$ en las propiedades finales del Relaxor PMB procesado por mezcla de polvos". Bol. Soc. Esp. Ceram. V. $43,3,653-657(2004)$

32. L. Lascano, A. C. Caballero, M. Villegas, C. Moure, J. F. Fernandez. "Conductividad eléctrica en corriente alterna de materiales cerámicos aurivillius de composición $\mathrm{PB}_{\mathrm{x}} \mathrm{Bi}_{4} \mathrm{Ti}_{3+\mathrm{x}} \mathrm{O}_{12+3 x^{\prime}} \mathrm{x}=0,1,2$ y 3". Bol. Soc. Esp. Ceram. V. $43,2,536-539$ (2004)

33. M. Algueró, B. Jiménez, C. Alemany, L. Pardo. “Temperature dependence of the electrical, mechanical and electromechanical properties of high sensitivity novel piezoceramics". Bol. Soc. Esp. Ceram. V. 43, 2, 540-543 (2004)

34. A. Lopez, R. E. Alonso. "Interacciones cuadripolares en $\mathrm{SrZrO}_{3}$ ". Bol. Soc. Esp. Ceram. V. 43, 3, 695-697 (2004)

35. M. Ramajo, E. Brozozowski, M. S. Castro. "Estudio del mecanismo de compensación de $\mathrm{BaTiO}_{3}$ dopado con $\mathrm{La}_{2} \mathrm{O}_{3}$ ". Bol. Soc. Esp. Ceram. V. 43, 3, 691-694 (2004)

36. J. C. Moreno, J. F. Fernandez, P. Ochoa, R. Ceres, L. Calderón, E. Rocon, J. L. Pons. "Aplicación de sensores piezoeléctricos cerámicos a la caracterización biomecánica”. Bol. Soc. Esp. Ceram. V. 43, 3, 668-673 (2004)
37. D. Mesonero, J."Comparación entre sistemas electrocerámicos de desplazamiento mecánico. Motores y actuadores piezoeléctricos". Bol. Soc. Esp. Ceram. V. 43, 4, 725-731 (2004)

38. H. Rodriguez, R. Ceres, L. Calderon, J. L. Pons. " Modelling of the travelling wave piezoelectric motor stator: an integrated review and new perspective". Bol. Soc. Esp. Ceram. V. 43, 3, 698-705 (2004)

39. M. A. De la Rubia, M. Peiteado, J. F. Fernández, A. C. Caballero. “Estudio de la región rica en $\mathrm{Bi}_{2} \mathrm{O}_{3}$ en el sistema binario $\mathrm{ZnO}-\mathrm{Bi}_{2} \mathrm{O}_{3}$ ". Bol. Soc. Esp. Ceram. V. $43,4,745-747(2004)$

40. H. Avila, A. M. Cruz, M. Villegas, A. C. Caballero, J. E. Rodriguez-Paez. "Estudio comparativo de dos metodos de sintesis para la obtención de polvos cerámicos de $\mathrm{ZnO}-\mathrm{Pr}_{2} \mathrm{O}_{3}-\mathrm{CoO}^{\prime}$. Bol. Soc. Esp. Ceram. V. 43, 4, 740-744 (2004)

41. Fernandez Hevia, A. C. Caballero, J. De Frutos, J. F. Fernandez. “Espectroscopia de admitancias de banda ancha aplicada al control microestructural de propiedades eléctricas en varistores cerámicos". Bol. Soc. Esp. Ceram. V. 43, 3, 674-678 (2004)

42. M. Valant, D. Suvorov. "New Generation of LTCC Materials". Bol. Soc. Esp. Ceram. V. 43, 3, 634-639 (2004)

43. Yanwei M. A., M. Bahout, O. Peña, P. Durán, C. Moure. “Magnetic properties of $(\mathrm{Co}, \mathrm{Ni}, \mathrm{Mn})_{3} \mathrm{O}_{4}$ spinels". Bol. Soc. Esp. Ceram. V. 43, 3, 663-667 (2004)

44. J. Fontcubierta, LL. Balcells, J. Navarro, D. Rubi, B. Martinez, C. Frontera, J. L. García-Muñoz, M. Lacaba, A. M. González, C. Fronies, A. Calleja, LL. Aragones. "Magnoteresistive ceramics. Recent progress: from basic understanding to applications". Bol. Soc. Esp. Ceram. V. 43, 627-633 (2004)

45. T. Hernandez, I. Gómez, J. M. Greneche, F. Plazaola, J. M. Barandiaran. "Propiedades magnéticas y espectroscopia mössbauer en compuestos pervskita doble $\mathrm{Sr}_{2}\left(\mathrm{Fe}_{1-\mathrm{x}} \mathrm{Sc}_{\mathrm{x}}\right) \mathrm{ReO}_{6}(\mathrm{x}=0.05,0.1)$ ". Bol. Soc. Esp. Ceram. V. 42, 5, 317-321 (2003)

46. O. Peña, D. Gutierrez, P. Durán, C. Moure. «Magnetic properties of manganese-site substituted $\mathrm{Y}(\mathrm{Cu}, \mathrm{Mn}) \mathrm{O}_{3}{ }^{\prime \prime}$. Bol. Soc. Esp. Ceram. V. 43, 4, 732735 (2004)

47. O. Peña, K. Ghanimi, C. Moure, D Gutiérrez, P. Durán. «Spin reversal in $\mathrm{Gd}(\mathrm{Me}, \mathrm{Mn}) \mathrm{O}_{3}(\mathrm{Me}=\mathrm{Co}, \mathrm{Ni}) »$. Bol. Soc. Esp. Ceram. V. 43, 3, 706-709 (2004)

48. E. Rossinyol, J. Arbiol, F. Peiró, A. Cornet, J. R. Morante, V. Brinzari, G. Korotcenkov, V. Golovanov. «Evolución de la morfología y facetaje de nanoestructuras de $\mathrm{SnO}_{2}$ crecidas por pirólisis en fase aerosol sobre sustratos de vidrio». Bol. Soc. Esp. Ceram. V. 43, 2, 510-513 (2004)

49. M. A. Ponce, C. M. Aldao, M. S. Castro. "Adsorción de oxígeno en sensores de película gruesa de $\mathrm{SnO}_{2}$ dopados con Pd". Bol. Soc. Esp. Ceram. V. 43, 3, 686-690 (2004)

50. O. Casals, M. Haffar, B. Barcones, A. Romano, C. Serre, A. Pérez, J. R Morante, P. Godignon, J. Montserrat, J. Millán. "Estudio de puertas catalíticas en sensores de gas en tecnologíade $\mathrm{SiC}^{\prime \prime}$. Bol. Soc. Esp. Ceram. V. 43, 2, 383-385 (2004)

51. I. G. Cano, M. A. Rodriguez. "Obtención de $\mathrm{Si}_{3} \mathrm{~N}_{4}$ mediante SHS". Bol. Soc. Esp. Ceram. V. 42, 2, 89-92 (2003)

52. J. Carretero, M. A. Sainz, S. Serena, A. Caballero. “Obtención de circonas estabilizadas (Ca, Mg-PSZ) nanocristalinas a partir de mezclas de dolomita y circona monocíclica mediante molienda de alta energía". Bol. Soc. Esp. Ceram. V. $42,5,303-310$ (2003)

53. C. Gomez, J. M. Albella, F. Ojeda, F. J. Martí. “Síntesis de materiales cerámicos mediante técnicas químicas en fase vapor (CVD)". Bol. Soc. Esp. Ceram. V. 42, 1, 27-31 (2003)

54. E. Fernandez, P. Sanchez, M. Panizza, V. Sánchez, J. M. Gallardo, G. Busca, E. Tejedor, C. Resini. "Influencia de la adición de galio en las propiedades estructurales y texturales de óxidos de $\mathrm{Mn} \mathrm{y} \mathrm{Al}^{\prime}$. Bol. Soc. Esp. Ceram. V. 43 2, 132-134 (2004)

55. M. D. Alcalá, C. Real, J. M. Criado. "Influencia de los reactivos y de las condiciones experimentales en la síntesis carbotérmica de nitruro de silicio". Bol. Soc. Esp. Ceram. V. 43, 6, 933-938 (2004)

56. A. Hernández-Jimenez, F. Sanchez-Bajo, A. L. Ortiz, F. L. Cumbrera "Determinación de la composición de fases en circona mediante un procedimiento polimórfico simple". Bol. Soc. Esp. Ceram. V. 43, 1, 23-25 (2004)

57. A. Morales, A. Bravo, A. Domínguez, M. Jiménez. "Microestructura de $\mathrm{Al}_{2} \mathrm{O}_{3}$ TZP codopado con $\mathrm{Fe}_{2} \mathrm{O}_{3}$ y TiO fabricado por reacción (RBAO)". Bol. Soc. Esp Ceram. V. 42, 1, 5-8 (2003)

58. M. P. Villar, L. Lago-Duport, T. García. "Comportamiento de mullitas a alta temperatura: Estudio mediante Difracción de Rayos X". Bol. Soc. Esp. Ceram. V. $43,2,135-137$ (2004)

59. A. Morales-Rodriguez, A. Bravo-León, M.Jimenez-Melendo, A. DomínguezRodriguez. "Comportamiento plástico a alta temperatura de $\mathrm{Al}_{2} \mathrm{O}_{3}$ codopado con $\mathrm{CuO}_{\text {y TiO}}$ fabricado por reacción". Bol. Soc. Esp. Ceram. V. 42, 4, 229-233 (2003)

60. G. Fargas Ribas, D. Casellas Padró, L. M. Llanes Pitach, M. Anglada Gomila. "Resistencia al choque térmico de Y-TP con fisuras Palmqvist". Bol. Soc. Esp. Ceram. V. 42, 1, 9-14 (2003)

61. A. Feder, L. Llanes y M. Anglada. "Efecto de la nitruración en la degradación hidrotérmica de circona tetragonal policristalina estabilizada con $\mathrm{Y}_{2} \mathrm{O}_{3}$ ". Bol. Soc. Esp. Ceram. V. 43, 1, 47-52 (2004) 
62. A. Tallaire, F.J. Oliveira, A. J. S. Fernandez, F. M. Costa, M. Belmonte, R. F. Silva. " $\mathrm{Si}_{3} \mathrm{~N}_{4}$ recubierto con diamante CVD mediante filamento caliente y plasma generado por micoondas". Bol. Soc. Esp. Ceram. V. 43, 2, 473-476 (2004)

63. A. Ureña, J. Rams. M. D. Escalera y M. Campo. "Estudia de la protección del refuerzo de partículas de $\mathrm{SiC}$ mediante barreras activas por sol-gel en materiales compuestos de matriz de “.Bol. Soc. Esp. Ceram. V. 43, 2, 397-400 (2004)

64. F. M. Morales, J. Ramirez, C. Fernandez, L. Barbadillo, J. Piqueras, D. Araujo, S. I. Molina, R. García. "Estudio por microscopía y espectroscopia de inflarojos de capas de $\mathrm{SiC}$ obtenidas mediante carburización de obleas de $\mathrm{Si}^{\prime \prime}$. Bol. Soc. Esp. Ceram. V. 43, 2, 363-366 (2004)

65. T. E. Novelo, O. Ceh, M. A. Pecha-Canul, A. I. Oliva. "Cinética de formación del $\mathrm{Al}_{2} \mathrm{O}_{3}$ en capas de aluminio estudiada mediante mediciones eléctricas" Bol. Soc. Esp. Ceram. V. 43, 4, 801-804 (2004)

66. J. M. Benito, A. Conesa, M. A. Rodríguez. "Membranas cerámicas. Tipos, métodos de obtención y caracterización". Bol. Soc. Esp. Ceram. V. 43, 5, 829842 (2004)

67. P. Prádanos, L. Palacio, A. Hernandez, M. Vilaseca, M. Vilaseca, J. Coronas, J. Santamaría. "Estudio mediante afm de estructuras de silicalita para la separación de gases". Bol. Soc. Esp. Ceram. V. 43, 1, 19-22 (2004)

68. H. El Kinani, L. Yahyaoui-Azami, M. Rafiq, E. Rodríguez-Castellón, J. Benavene. "Caracterización estructural y electroquímica de una membrana compuesta de $\mathrm{TiO}_{2}$ y de su soporte poroso basado en arcillas naturales". Bol. Soc. Esp. Ceram. V. 43, 1, 38-41 (2004)

69. J. C. Diez, J. I. Peña, V. M. Orera, M. Sierra. "Resolidificación con láser de barreras térmicas de circona depositadas por proyección térmica plasma (APS)" Bol. Soc. Esp. Ceram. V. 43, 6, 925-928 (2004)

70. A. Díaz, F. Guiberteau, R. Caruso. "Influencia del tratamiento térmico sobre el espesor y porosidad de películas delgadas de $\mathrm{ZrO}_{2}$ obtenidas por la ruta sol-gel". Bol. Soc. Esp. Ceram. V. 43, 2, 477-479 (2004)

71. B. Ferrari, J. L. Rodríguez, E. Rojas, R. Moreno. "Conformado por vía coloidal de recubrimientos en a cara interna de un tubo de acero". Bol. Soc. Esp. Ceram. V. $43,2,501-505$ (2004)

72. R. I. Merino, J. I. Peña, V. M. Orera, A. Karrea y A. J. Sanchez Herencia. "Resolidificación superficial de eutécticos $\mathrm{Al}_{2} \mathrm{O}_{3}$ YSZ asistida por láser". Bol. Soc. Esp. Ceram. V. 43, 5, 855-862 (2004)

73. A. J. Ramirez del Valle, S. Palanco, J. J. Laserna, J. Pascual Cosp. “Corte de placas solares con láser. Estudio de modificaciones microestructurales". Bol. Soc. Esp. Ceram. V. 42, 3, 151-155 (2003)

74. I. Cañadas, D. Martínez, J. Rodríguez. "Tratamiento térmico de materiales en el horno Solar de la PSA: líneas actuales de actividad". Bol. Soc. Esp. Ceram. V. 43, 2, 591-595 (2004)

75. J. J. Coronel, R. Janssen, N. Claussen. “Conformado de piezas cerámicas a partir de suspensiones parafínicas cargadas con polvos". Bol. Soc. Esp. Ceram. V. $43,5,843-848(2004)$

76. P. N. de Aza, Z. B. Luklinska. "Efecto de la microestructura sobre la bioactividad de dos materiales vitrocerámicos del sistema $\mathrm{CaSiO}_{3}-\mathrm{ZrO}_{2}$ ". Bol. Soc. Esp. Ceram. V. 42, 2, 101-106 (2003)

77. G. Vargas. A. Muñoz, J. Mendez, M. Méndez, P. Omondragón. “Deposición electroforética de una porcelana dental sobre acero inoxidable austenítico $304^{\prime \prime}$. Bol. Soc. Esp. Ceram. V. 42, 1, 21-26 (2003)

78. D. A. Cortés, A. Medina, J. C. Escobedo, S. Escobedo, M. A. López. “Uso de sistemas bioactivos en la formación de apatita en la superficie de aleaciones base cobalto". Bol. Soc. Esp. Ceram. V. 43, 5, 863-868 (2004)

79. V. Amigó, A. Vicente, F. Romero, y A. Paolini. "Influencia del tratamiento HIP en la distribución de los carburos en prótesis Co-Cr-Mo". Bol. Soc. Esp. Ceram. V. 43, 2, 573-577 (2004)

80. R. Ferré, B. Garrido, P. Pellegrino, C. García, J. R. Morante. “Nanocristales de silicio en matriz de $\mathrm{SiO}_{2}$ para aplicaciones fotónicas". Bol. Soc. Esp. Ceram. V. 43, 2, 390-303 (2004)

81. J. Cembrero, M. Oerales, M. Mollar, B. Marí. “Obtención de columnas de $\mathrm{ZnO}^{\prime \prime}$. Bol. Soc. Esp. Ceram. V. 42, 6, 379-387 (2003)

82. J. A. Picas, A. Forn, L. Ajdelsztajn. J. Schoenung. “Morfología de los óxidos superficiales en los recubrimientos convencionales y nanocristalinos de NiCrAlY sometidos a alta temperatura". Bol. Soc. Esp. Ceram. V. 43, 2, 518520 (2004)

83. C. Lorenzo-Martí, D. Gomez-García, A. Muñoz-Bernabé, A. DomínguezRodriguez. "Fluencia a alta temperatura de policristales con tamaño de grano nanometrico de YTZP dopados con diferentes cantidades de fase vítrea", Bol. Soc. Esp. Ceram. V. 43, 2, 521-523 (2004)

84. B. Vazquez, A. Caballero, P. Pena. "Estudio del campo primario del $\mathrm{Al}_{2} \mathrm{O}_{3}$ en el sistema cuaternario $\mathrm{Al}_{2} \mathrm{O}_{3}-\mathrm{CaO}-\mathrm{MgO}-\mathrm{SiO}_{2}$ ". Bol. Soc. Esp. Ceram. V. 43, 1, 16-18 (2004)

85. A. H. de Aza, P. Pena, S. de Aza, M. Raigón, J. Gómez-Millán, C. Domínguez. "Estudio post-mortem de un hormigón refractario colocado en la línea de escorias de una cuchara de acería". Bol. Soc. Esp. Ceram. V. 42, 6, 375-378 (2003)

86. J. M. Rivas Mercury, A. H. de Aza, X. Turrillas, P. Pena. "Hidratación de los cementos de aluminatos de calcio (Parte I)". Bol. Soc. Esp. Ceram. V. 42, 5, 269-276 (2004)
87. J. M. Rivas Mercury, A. H. de Aza, X. Turrillas, P. Pena. "Hidratación de los cementos de aluminatos de calcio. Parte II: Efecto de las adiciones de sílice y alumina". Bol. Soc. Esp. Ceram. V. 42, 6, 361-368 (2003)

88. C. R. Ferrari, J. A. Rodrígues. "Microstructural features of alumina refractories with mullite-zirconia aggregates". Bol. Soc. Esp. Ceram. V. 42, 1, 15-20 (2003)

89. A. H. de Aza, X. Turrillas, J. L. Rodríguez, P. Pena. "Estudio del proceso de sinterización reactiva en sistemas con dolomita mediante termodifractometría de neutrones". Bol. Soc. Esp. Ceram. V. 43, 1, 12-15 (2004)

90. A. P. Fonseca, J. Baptista. "Efecto de la estequiometria y de la temperatura de cocción en el desarrollo de la fase $\mathrm{Al}_{2} \mathrm{TiO}_{5}{ }^{\prime \prime}$. Bol. Soc. Esp. Ceram. V. 42, 2, 65-68 (2003)

91. G. Vargas, F. Vazquez, J. Lopez, J. Mendez, M. Mendez, P. Pena. “Espumado de mezclas de silicato de sodio-wollastonita por microondas". Bol. Soc. Esp. Ceram. V. 43, 1, 71-74 (2004)

92. V. Correcher, L. M. Robredo, F. J. Valle. Fuentes, P. López-Arce, J.GarciaGuinea. "Caracterización de la curva de termoluminiscencia del circón a partir de su contenido radiactiva y del análisis térmico diferencial". Bol. Soc. Esp. Ceram. V. 42, 6, 369-373 (2003)

93. V. Correcher, F. J. Valle Fuentes y J. García- Guinea. “Espectros de emisión de radioluminiscencia y termoluminiscencia de una leucita de Monte Somma (Italia). Bol. Soc. Esp. Ceram. V. 43, 4, 919-924 (2004)

94. J. Garcia-Guinea, J. F. Pitalua, V. Correcher, L. Sanchez-Muñoz, F. J. Valle Fuentes, P. Lopez-Arce. Bol. Soc. Esp. Ceram. V. 43, 2, 115-118 (2004)

95. M. F. Gazulla, M. P. Gómez, E. Mondort, M. Orduña. "Interferencias en la caracterización de residuos cerámicos mediante el ensayo de inhibición de la luminiscencia". Bol. Soc. Esp. Ceram. V. 43, 6, 909-914 (2004)

96. J. García-Guinea, D. Hole, A. Finch, V. Correcher, F.J. Valle-Fuentes, N. Can, P. D. Townsend. "Ionoluminiscencia de silicatos de utilización en cerámica a temperaturas criogenicas". Bol. Soc. Esp. Ceram. V. 43, 5, 877-882 (2004)

97. P. Martin, A. Moroño, E. R. Hodgson. "Cuarzo KU1 de alta resistencia a la radiación". Bol. Soc. Esp. Ceram. V. 43, 2, 452-454 (2004)

98. V. Correcher, y J. García-Guinea. "Cambios en las propiedades luminiscentes de un feldespato potásico inducidos por efecto térmico y exposición a infrarrojo". Bol. Soc. Esp. Ceram. V. 43, 1, 5-7 (2004)

99. J. Garcia-Guinea, F. Pitalua, V. Correcher, P. López-Arce. “Observación de deshidrataciones y contracciones de una arcilla por difracción de rayos $\mathrm{x} y$ microscopia electrónica a temperatura y humedad controladas". Bol. Soc. Esp. Ceram. V. 43, 1, 26-29 (2004)

100. M. A. Bustillo, E. García Romero. "Arcillas fibrosas anómalas en encostramientos y sedimentos superficiales: características y génesis (Esquivias, Cuenca de Madrid)". Bol. Soc. Esp. Ceram. V. 42, 5, 289-297 (2003)

101. M. Vicente. M. Llusar, G. Monrós. "Caracterización cerámica de los sedimentos de la Albufera de Valencia". Bol. Soc. Esp. Ceram. V. 42, 3, 145150 (2003).

102. M. Vazquez, J. Jimenez Millan, C. Sanchez-Jimenez, J. Parras. “Composición y propiedades cerámicas de las pizarras de la Zona Centro Ibérica del Macizo Ibérico Meridional (Norte de Jaén, España)". Bol. Soc. Esp. Ceram. V. 42, 4, 215-221 (2003)

103. C. Marcos, A. Ruiz-Conde, A. Argüelles, P. J. Sánchez-Soto, A. García, J. A. Blanco. "Nuevos avances en la formación de fases interestratificadas durante al proceso de deshidratación-rehidratación de vermiculitas-Mg: Influencia de la presión de vacío, temperatura y composición". Bol. Soc. Esp. Ceram. V. 43, 2, 138-140 (2004)

104. V. Rives, M. Del Arco, O. Prieto. “Birnesitas obtenidas mediante cambio iónico. Evolución estructural con la calcinación". Bol. Soc. Esp. Ceram. V. 43 , 2, 142-147 (2004)

105. C. Belver. M. A. Bañares, M. A. Vicente. "Materiales con propiedades tecnológicas obtenidos por modificación química de un caolín natural". Bol. Soc. Esp. Ceram. V. 43, 2, 148-154 (2004)

106. J. Bastida, C. Bolós, P. Pardo, F. J. Serrano. "Análisis microestructural por DRX de $\mathrm{CaO}$ obtenido a partir de carbonato cálcico molido (CCM)". Bol. Soc. Esp. Ceram. V. 43, , 80-83 (2004)

107. M. Popa, J. M. Calderón Moreno "Cristalización de vidrios ricos en sílice preparados mediante sol-gel en el sistema alúmina-circona-sílice". Bol. Soc. Esp. Ceram. V. 43, 1, 63-66 (2004)

108. A. Morales, M. C. Bautista, D. Cáceres. "Recubrimientos antirreflectantes de sílice sobre vidrio producidos por sol-gel". Bol. Soc. Esp. Ceram. V. 43, 2, 455-546 (2004)

109. E. F. Montero, M. A. García, M. A. Villegas, J. Llopis. "Estudio de las propiedades ópticas de recubrimientos Sol-Gel dopados con fluoresceína en función de la concentración y del $\mathrm{pH}^{\prime \prime}$. Bol. Soc. Esp. Ceram. V. 43, 1, 8-11 (2004)

110. I. García Perulero, S. Murcia Mascarós, J. Rubio, J. L. Oteo. "Síntesis y caracterización de materiales híbridos orgánico-inorgánicos de APS/PDMS". Bol. Soc. Esp. Ceram. V. 42, 6, 389-396 (2003)

111. L. Téllez, F. Rubio, R. Peña Alonso, J. Rubio. “Seguimiento por espectroscopia infrarroja (FT-IR) de la copolimerización de TEOS (tetraetilortosilicato) y PDMS (polidimetilsiloxano) en presencia de tbt (tetrabutiltitanio)". Bol. Soc. Esp. Ceram. V. 43, 5, 883-890 (2004)

112. M. A. Diaz Diez, A. Macias Garcia, G. Silvero, R. Gordillo y R. Caruso. "Determinación de la estructura molecular de geles mediante cálculos computacionales". Bol. Soc. Esp. Ceram. V. 43, 2, 480-482 (2004) 
113. J. E. Rodriguez Paez, A. Mafla, G. Andrade, A. Durán. "Modificación química del precursor de titanio para obtener soles estables de silice-titania: Uso de acetilacetona". Bol. Soc. Esp. Ceram. V. 43, 1, 53-55 (2004)

114. C. Lara, M. J. Pascual, A. Durán. "Sellos vítreos y vitrocerámicos para pilas de combustible de óxido sólido (SOFC)". Bol. Soc. Esp. Ceram. V. 42, 2, 133144 (2003)

115. S. Gabaldón, S. López, J. B. Carda. “Legislación y gestión medioambiental en la producción de baldosas cerámicas". Bol. Soc. Esp. Ceram. V. 42, 4, 169179 (2003)

116. J. M. Villora, P. Callejas, M. F. Barba. "Ingluencia del procesamiento como adsorbente de iones en aguas industriales". Bol. Soc. Esp. Ceram. V. 43, 1, 75-79 (2004)

117. S. Hernandez, R. Juan, X. Querol, A. Alastuey, P. Ferrer, J. M. Andrés "Activación de cenizas volantes para retención de gases contaminantes". Bol. Soc. Esp. Ceram. V. 42, 1, 33-37 (2003)

118. A. M. Urtiaga, E. D. Gorri, C. Casado, I. Ortiz. "Funcionamiento de una membrana de zeolita 4-A comercial en la deshidratación de disolventes industriales mediante pervaporación". Bol. Soc. Esp. Ceram. V. 43, 1, 35-37 (2004)

119. J. F. Betancur, Y. B. Restrepo,, P. Abad, M. Arias. "Inmovilización de lactobacillus helveticus en soportes vítreos obtenidos por tecnología sol-gel". Bol. Soc. Esp. Ceram. V. 42, 2, 79-83 (2003)

120. A. M. García. J. M. Villora, D. A. Moreno, C. Ranninger, P. Callejas, M. F. Barba. "Aplicación de un material vitrocerámico a la biorremediación de metales pesados". Bol. Soc. Esp. Ceram. V. 43, 1, 59-62 (2004)

121. P. Benito, F. M. Labajos, V. Rives. "Incidencia de la radiación microondas en la cristalinidad de materiales laminares". Bol. Soc. Esp. Ceram. V. 43, 1, 56-58 (2004)

122. S. H. Jazayeri, A. Salem, G. Timellini, E. Rastelli, A. Albertazzi. "Influence of nepheline syenite and milling time on the rheological properties of a porcelain stoneware tile slip". Bol. Soc. Esp. Ceram. V. 42, 4, 209-213 (2003)

123. M. Romero. J. M. Rincon, A. R. Boccaccini. "Coeficiente de fragilidad como medida más representativa de la resistencia a la abrasión de pavimentos cerámicos" Bol. Soc. Esp. Ceram. V. 42, 3, 163-167 (2003)

124. L. Escoda, M. Lledo, J. J. Suñol, P. Roura, J. Carda. "Estudio sobre la resistencia química de baldosas cerámicas no-esmaltadas para pavimentos industriales". Bol. Soc. Esp. Ceram. V. 42, 2, 85-88 (2003)
125. O. Rodriguez, W. Romero, J. A. Vega, S. Esguerra. "Influencia térmica e higroscópica en la permitividad relativa de material cerámico crudo utilizado en el proceso de fabricación de baldosas para piso". Bol. Soc. Esp. Ceram. V. 42, 2, 205-207 (2003)

126. H. Camacho, M. E. Fuentes, L. Fuentes, A. García, A. Pérez. "Evolución de la distribución de tensiones en un cuerpo cerámico durante de cocción. Parte 1: Planteamiento de los problemas". Bol. Soc. Esp. Ceram. V. 42, 5, 283-288 (2003)

127. H. Camacho, M. E. Fuentes, L. Fuentes, A. Garcia, A. Pérez. "Evolución de la distribución de tensiones en un cuerpo cerámico durante la cocción. Parte 2: Cálculo de los perfiles". Bol. Soc. Esp. Ceram. V. 42, 6, 353-359 (2003)

128. B. Segura, E- Vallada, C. Maroto, R. Ruiz. "Análisis del sistema de operaciones en empresas del sector cerámico español". Bol. Soc. Esp. Ceram. V. 43, 6, 929-932 (2004)

129. F. Lucas, A. Belda. F. J. Torres, J Alarcón. "Estudio y caracterización de vidriados vitrocerámicos basados en piroxeno". Bol. Soc. Esp. Ceram. V. 43, 5, 849-854 (2004)

130. TZ. Dimitriv, K. Georgieva, S. Vassilev. "Study of ceramic pigments from the $\mathrm{ZrO}_{2}-\mathrm{SiL}_{2}-\mathrm{Fe}_{2} \mathrm{O}_{3}$ system". Bol. Soc. Esp. Ceram. V. 42, 4, 235-237 (2003)

131. R. Pozas, M. P. Morales, C. J. Serna, M. Ocaña. “Acicular iron nanoparticles protected against sintering with aluminium oxide". Bol. Soc. Esp. Ceram. V. 43, 4, 796-800 (2004)

132. S. Sorli, M. Llusar, J. Calbo, P. Benet, J. A. Badenes, M. A. Tena, G. Monrós. "Caracterización eléctrica de disoluciones sólidas $(\mathrm{Cr}, \mathrm{V}, \mathrm{Ti}) \mathrm{O}_{2}$ con estructura rutilo". Bol. Soc. Esp. Ceram. V. 43, 4, $792-795$ (2004)

133. R. Fort. M. Alvarez de Buergo, M. C. López de Azcona, F. Mingarro, M. J. Varas, J. Soriano. "Caracterización de la Fábrica de Ladrillo del Palacio del Infante Don Kuis, Boadilla del Monte, Madrid". Bol. Soc. Esp. Ceram. V. 43, 2, 578-582 (2004)

134. A. J. Polvorinos, del Tío, V. Flores, M. A. Tabales, M. J. Gernandez "Caracterización y tecnología de materiales cerámicos romanos de los ss. I a III d.c. procedentes del Hospital de las Cinco Llagas de Sevilla". Bol. Soc. Esp. Ceram. V. 42, 2, 93-99 (2003)

135. M. Seco Alvarez, A. Ruiz Conde, M. A. Villegas, P. J Sanchez Soto. “E vidrio en la civilización egipcia. Descripción de piezas de vidrio del tipo incrustaciones de una colección de Universidad de Sevilla". Bol. Soc. Esp. Ceram. V. 42, 4, 223-228 (2003)

136. M. Bordons, A. Felipe, I. Gomez. “ Revistas españolas con factor de impacto en el año 2000”. Rev. Esp. Doc. Cient. V. 21, 1, 49-73 (2002 